\title{
Carbapenem Resistant Enterobacteriaceae in Africa
}

Bashar Haruna Gulumbe ${ }^{*}[0$
Olumide Ajibola 2
1Department of Microbiology, Federal
University Birnin-Kebbi, Birnin Kebbi,
Kebbi State, Nigeria
2First Technical University, Ibadan,
Ibadan, Oyo State, Nigeria
"email: hgbashar@gmail.com
Keywords:
Africa
Antibiotic resistance
Carbapenem
Enterobacteriaceae

\begin{abstract}
Carbapenems are regarded as unique among the $\beta$-lactam antibiotics due to their broad spectrum of activity and ability to resist $\beta$-lactamase hydrolysis. Carbapenems are the only $\beta$-lactam antibiotics with efficacy in severe infections caused by extended-spectrum betalactamase (ESBL) producing bacteria. However, recent reports of carbapenem resistance particularly among members of Enterobacteriaceae that are responsible for diseases such as gastrointestinal infections, septicemia, pneumonia, meningitis, peritonitis as well as urinary tract infections, call for concerns. In Africa, the problem of carbapenem-resistant Enterobacteriaceae (CRE) is aggravated by factors such as the high rate of infections, poor diagnostic tools, sub-optimal disease surveillance, and abuse of antibiotics. Besides, the problem of CRE in Africa is understudied. This review distills available literature on the spread of CRE in Africa, CRE genes in circulation, and the need to pay attention to this emerging threat to lives in developing countries.
\end{abstract}

Received: April 19th 2020

Accepted: May 17th 2020

Published: May 21 2020 (http://creativecommons.org/licenses/by-sa/4.0/). DOI: https://doi.org/10.33084/bjop.v3i2.1356

\section{INTRODUCTION}

The development of resistance to antimicrobials, which presents a major global public health threat has been evolving rapidly in recent years and spread almost all over the world (Codjoe \& Donkor, 2017; Ventola, 2015). Globally, annual mortality rate due to antimicrobial resistance (AMR) is estimated at 700,000 and by the year 2050 AMR is estimated to claim up to 10 million lives with an annual economic burden of US \$100 trillion (Tadesse et al., 2017). In Africa, paucity of CRE data as presented in Figure $\mathbf{1}$ has hindered estimates of the magnitude of the problem, in addition to increased cases of treatment failure that has resulted in grave socioeconomic indices for the affected population (Kariuki \& Dougan, 2014).

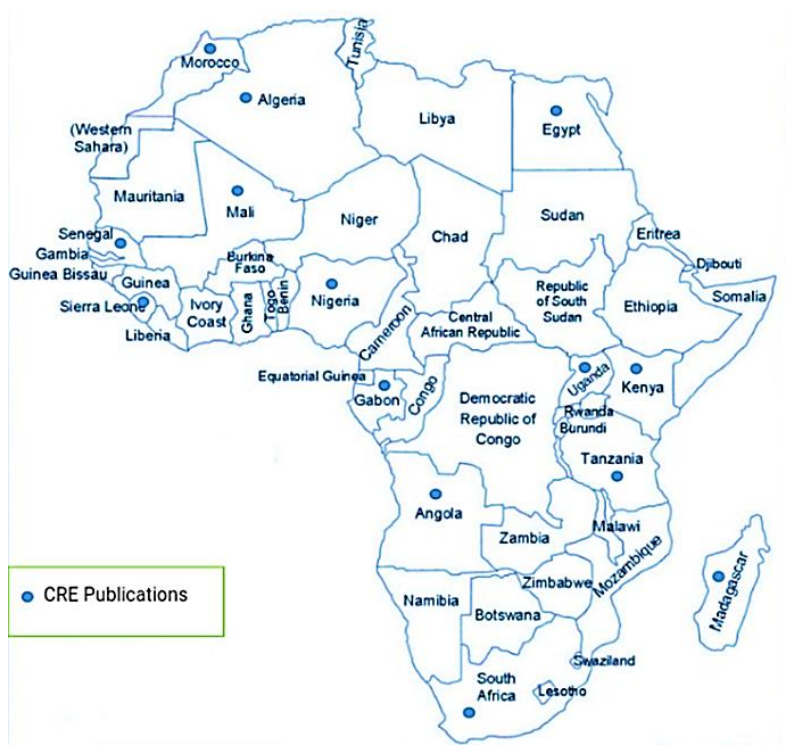

Figure 1. Countries with CRE published research in Africa

Carbapenems are generally regarded as the most effective type of antibiotics because of their proven effect against bacterial infections including those caused by antibiotic-resistant enterobacteriaceae (Iovleva \& Doi, 
2017). Unfortunately, resistance to carbapenems have been reported. The first case of carbapenem resistance was detected from Aeromonas hydrophila isolate in 1980s in Japan and subsequent cases were reported in London, UK (1982) from Serratia marcescens, California, USA (1984) and France (1990), both from Enterobacter cloacae (Codjoe \& Donkor, 2017).

Enterobacteriaceae, are a group of rod-like Gramnegative bacteria, the most commonly encountered bacteria in clinical samples and may be responsible for approximately $80 \%$ of clinically significant Gramnegative bacilli and $50 \%$ of all clinically significant bacteria (Perovic et al., 2016). In Africa, the problem is inflamed by suboptimal disease surveillance and healthcare system, ineffective infection control policies, indiscriminate use of antibiotics, poor diagnostic tools and lack of effective antibiotic stewardship programs in most parts of the continent (Manyi-Loh et al., 2018). In this review, we provide an up-to-date synthesis of CRE data in Africa, highlighting the need for more research within the continent on the surveillance of CRE genes.

\section{COMMUNITY AND HOSPITAL ACQUISITION OF CRE}

Infections due to CRE can be acquired in both clinical and community settings and are extremely difficult to treat. CRE, particularly Escherichia coli and Klebsiella pneumoniae, were relatively uncommon causes of hospital acquired infections until about two decades ago, and have since doubled in prevalence in recent years (Kelly et al., 2017). Isolation of patients helps reduce patient-to-patient transmission of CRE, and has led to a significant reduction of CRE infections in patients with confirmed CRE colonisation at the time of admission, whether symptomatic or not (Magiorakos et al., 2017). The number of individuals that develop infection after colonisation remains unclear (Dortet et al., 2014). In a systematic review where 1,806 hospitalised patients identified as colonised with CRE at the time of admission were studied, only $299(16.5 \%)$ were found to develop infection (Tischendorf et al., 2016). Evidence suggests that longterm hospitalisation plays a critical role in the dissemination of CRE. Therefore, early detection of CRE in patients admitted to health facilities may help mitigate institutional outbreaks and halt regional spread of CRE (Codjoe \& Donkor, 2017).

The dissemination of CRE in the community is largely through carriage in commensal microflora, which might go undetected unless disease symptoms manifest (Kumarasamy et al., 2010). In poor communities with limited health facilities in Africa, even when symptoms develop, limited diagnostic and treatment options continues to promote dissemination of CRE among the population in affected communities (Maphumulo \& Bhengu, 2019).

The link between community and healthcare acquisition of CRE has been previously described by Dortet et al. (2014) who reported that New Delhi metallobetalactamase (NDM)-producing microorganisms isolated with high frequency in healthcare facilities and environmental niches disseminated into the community through patient transfer. Individuals who have a history of frequent exposure to CRE in a healthcare facility may easily spread CRE within the communities in Africa. Reports reveal that bacteria carrying the NDM enzyme may find its way outside the boundary of hospital settings into community water and sewage environments (Codjoe \& Donkor, 2017).

\section{CARBAPENEMS}

Carbapenems differ from penicillins and cephalosporins in the chemical structure of their $\beta$-lactam ring (Hawkey \& Livermore, 2012). Carbapenem refers to the $4: 5$ fused 
ring lactam of penicillins with a double bond between C2 and C-3, but at C-1 position, sulphur is substituted with carbon (Papp-Wallace et al., 2011). The hydroxyethyl side chain of carbapenems is a radical departure from the regular structure of penicillins and cephalosporins, all of which have an acylamino substituent on the $\beta$-lactam ring; the stereochemistry of this hydroxyethyl side chain is a defining feature of carbapenems and is important for their high potency (Hawkey \& Livermore, 2012). Additionally, this unique structure of carbapenems gives them protection against most $\beta$-lactamases such as metallo- $\beta$-lactamase (MBL) and ESBL, hence making them one of the last antibiotics of resort for treatment of antibiotic resistant infections (Codjoe \& Donkor, 2017; Knapp \& English, 2001). The carbapenems include imipenem, meropenem, doripenem, ertapenem, panipenem and biapenem.

As $\beta$-lactam antibiotics, carbapenems have a penicillinlike mode of action which kill bacteria by inhibiting cell wall synthesis (Hawkey \& Livermore, 2012). Carbapenems penetrate Gram-negative bacteria via outer membrane proteins (e.g. porins). The activity of carbapenems is initiated by penetrating the bacterial cell wall and binding to enzymes known as penicillin binding proteins (PBPs) through their active site (the $\beta$ lactam ring). Carbapenems traverse the periplasmic space, where they acylate enzymes (transglycolases, transpeptidases, and carboxypeptidases) that catalyse bacterial cell wall (Peptidoglycan) synthesis (Codjoe \& Donkor, 2017). Peptidoglycan synthesis involves simultaneous formation and autolysis, hence when formation is inhibited, autolysis continues leading to cell death. Carbapenems have a unique ability to bind multiple peptidoglycan synthesising enzymes (Hawkey \& Livermore, 2012). Carbapenems also have a broad spectrum of activity and are indicated majorly for the treatment of serious conditions such as intra-abdominal infections, complicated urogenital infections and mixed bacterial infections that are resistant to other beta-lactam antibiotics (Zhanel et al., 2007).

\section{MOLECULAR EPIDEMIOLOGY OF CRE IN AFRICAN ISOLATES}

Carbapenem hydrolysing enzymes identified in enterobacteriaceae are classified into Ambler classes A (actively hydrolyse carbapenems and are partially inhibited by clavulanic acid), B (which are mainly in the class of $\beta$-lactamases with the ability to hydrolyse carbapenems but are susceptible to inhibition by EDTA and $\mathrm{D}$ (serine- $\beta$-lactamases which are poorly inhibited by EDTA or clavulanic acid) $\beta$-lactamases (Codjoe \& Donkor, 2017).

Globally, among enterobacteriaceae, blaNDM, blaVIM, and blaIMP are the most frequently identified carbapenem resistant genes (Sekyere et al., 2016). In the United States and South America, Class A carbapenemases like K. pneumoniae carbapenemases (KPC) and Guiana extended spectrum (GES) are the most prevalent CRE genes, whereas in Europe substantial cases of KPC, including Imipenem-resistant Pseudomonas (IMP) and Verona integron-encoded metallo- $\beta$-lactamases (VIM) have been reported. Class D types of carabapenemases which consist of Oxacillinhydrolysing (OXA)-48-like enzymes in enterobacteriaceae and other OXA variants are commonly distributed in the Mediterranean. In India and Pakistan, Class B type especially NDM are prevalent (Codjoe \& Donkor, 2017).

In Africa, although the exact molecular epidemiology of carbapenemases and their genetic environment are not well studied, the blaOXA-48, blaIMP, blaVIM, and blaNDM in Acinetobacter baumannii, K. pneumoniae, Enterobacter cloacae, Citrobacter spp. and E. coli, 
respectively are the dominating carbapenemase genes characterised till date (Sekyere et al., 2016).

In North Africa, carbapenem hydrolysing enzymes have been documented in countries such as Algeria, Tunisia, Morocco, Libya, and Egypt among enterobacteriaceae species. In Algeria, the first documented carbapenemase among enterobacteriaceae was VIM-19 enzyme in E. coli and K. pneumoniae (Rodriguez-Martinez et al., 2010). In Tunisia, Ktari et al. (2006) detected K. pneumoniae harbouring blaVIM-4 for the first time, which was coexpressed with blaCTX-M-15 and blaCMY-4. Similarly, samples collected from patients of armed conflict from Libya and tested in Europe, were positive for blaOXA-48 and blaOXA-23 in K. pneumoniae (ST101, ST147, ST383, and ECI). OXA-48 and NDM-1-producing enterobacteriaceae in environmental, clinical and community settings have been reported to be prevalent in Morocco (Sekyere et al., 2016). Most class D carbapenemases (OXA-48 and blaOXA-48) were detected in Egypt from K. pneumoniae and E. coli in clinical specimens (Bathoorn et al., 2013).

South Africa has published more articles on CRE than any other African country. Since 2012, the South African National Antimicrobial Resistance Reference Laboratory has reported a total of 1,618 carbapenem non-susceptible isolates from all specimen types. Of the 1,258 enterobacteriaceae identified, 1,043 (83\%) isolates were confirmed to have carbapenem resistant genes (Perovic et al., 2016). KPC-2 was first detected in South Africa in 2012 (Brink et al., 2012) and is the most frequently reported in South Africa. Others such as blaOXA-48-like genes, have been found in K. pneumoniae, and in rare cases, E. coli and E. cloacae, where they mediate multidrug resistance. The first documented case of a blaNDM-1 in South Africa was in 2011 from a 63-year-old patient (Lowman et al., 2011). In the same year, the first blaKPC case in South Africa
(blaKPC-2) reported from E. cloacae and K. pneumonia was also identified in K. pneumoniae (Brink et al., 2012).

In West Africa, Nigeria and Ghana are the leading countries in the region that have documented CRE (Sekyere et al., 2016). In Nigeria, a study reported carbapenemase prevalence of $33.5 \%$ in a hospital setting Yusuf et al. (2012), and Chika et al. (2014) reported 12.5\% and $15.4 \%$ carbapenemase production in E. coli and $K$. pneumoniae isolates. Molecular analysis of the genes responsible for resistance by Ogbolu \& Webber (2014) identified blaNDM, blaVIM, and blaGES among $P$. aeruginosa, Proteus spp., K. pneumoniae, and E. coli, respectively as the genes responsible for resistance in $\beta$ lactam antibiotic. Oladipo et al. (2018) reported a high rate of resistance to ertapenem (30\%), levofloxacin (20\%), and colistin sulphate $(4 \%)$ in E. coli isolated from clinical specimens which they suggested could be as a result of plasmid transfer of AMR genes.

In Ghana, a recent study involving 111 carbapenem resistant Gram-negative bacteria showed that none of the isolates harbour KPC genes. However, the carbapenemase genes identified were blaNDM-1, blaVIM-1, and blaOXA-48 in A. baumannii, Pseudomonas species, and K. pneumoniae respectively (Codjoe et al., 2019). In Uganda, a study by Okoche et al. (2015) found carbapenemase prevalence of $22.4 \%$ and $28.6 \%$ using phenotypic and genotypic tests. In a recent study in the same country, among 56 isolates positive for carbapenemase encoding genes, K. pneumoniae was the species with the highest number (52.2\%) and most prevalent genes were blaVIM (21,10.7\%), blaOXA-48 (19, 9.7\%), blaIMP (12, 6.1\%), blaKPC (10,5.1\%), and blaNDM1 (5, 2.6\%). In Kenya, carbapenemase gene was first reported in a K. pneumoniae isolate (ST14) harbouring an blaNDM-1 on a $120 \mathrm{kbIncA} / \mathrm{C}$ plasmid in 2011 (Poirel et al., 2011). In Tanzania, in a study with 227 isolates, $21.6 \%$ were found to harbour IMP genes (Mushi et al., 2014). 
Mushi et al. (2014) also documented blaIMP, blaVIM, blaOXA-48, and blaKPC in E. coli, K. pneumoniae, P. aeruginosa, and Salmonella in a collection of 80 out of 227 isolates in a five-year prospective study between 2007 and 2012.

\section{MECHANISM OF CARBAPENEM RESISTANCE AMONG \\ ENTEROBACTERIACEAE}

Carbapenem resistance among members of enterobacteriaceae has been reported to be on the increase globally (Okoche et al., 2015). Mechanisms of carbapenems resistance include release of $\beta$-lactamases, efflux pumps, and mutations that interfere with the expression and/or function of porins and PBPs. Combinations of these mechanisms can cause high levels of carbapenem resistance in bacteria (Robin et al., 2010). While some bacterial strains may possess intrinsic resistance, some others may possess genetic elements such as plasmids or transposons which produce carbapenem-destroying $\beta$-lactamases (Kieffer et al., 2016). The acquired carbapenemases in the Ambler class A group which $K$. pneumoniae carbapenemases predominate, are the commonest type of $\beta$-lactamase enzymes encountered globally (Moussounda et al., 2017; Mitgang et al., 2018). Other carbapenem resistance mechanisms include hyper-expression of AmpC gene or decreased permeability of the outer membrane due to porin loss coupled with the expression of AmpC enzymes or ESBLs (Sangare et al., 2017).

\section{CRE SURVEILLANCE IN AFRICA}

The World Health Organization (WHO) 2014 report on global surveillance reported information on carbapenem resistance among Klebsiella from 7 of 47 WHO Africa region countries, and previous reviews of the epidemiology of CRE and underlying genotypes have yielded limited information on the WHO Africa region. Since its first detection, many countries particularly in Africa have limited or no surveillance activities that are specific for the threat posed by KPC. In fact, based on available published data, only South Africa has a national routine surveillance program in Africa; the National Health Laboratories/National Institute of Communicable Diseases through the Antimicrobial Resistance Reference Laboratories in the country screen isolates from various regions of the country suspected of carbapenemase production and publishes regularly in order to track the trends and the burden of carbapenem resistance in the country (Papp-Wallace et al., 2011).

Laboratory personnel and facilities play a crucial role in identifying, mapping, quantifying and communicating AMR (Okeke, 2016). A number of techniques are in place for the detection of carbapenem-hydrolysing enzymes including, automated systems, disc diffusion, selective agar, modified Hodge test, synergy tests (such as E-tests or double disc tests), spectrometrics and molecular techniques (Codjoe \& Donkor, 2017). The use of matrixassisted laser desorption ionisation time-of-flight mass spectrometry (MALDI-TOF MS) as a new method of detecting resistance in bacteria from fresh positive blood cultures has been described with good results (Muggeo et al., 2018). Carbapenem resistant enterobacteriaceae are detectable by the MALDI-TOF MS technique, and results can be obtained within 4 to 5 hours. MALDI-TOF MS is highly sensitive (97.1\%), specific (98.9\%) and can distinguish between carbapenemase-producing and non-carbapenemase-producing strains (Muggeo et al., 2018). However, despite their high sensitivity and specificity, the deployment of these contemporary techniques in African settings has been limited by factors such as huge cost of equipment, consumables, reagents and lack of skilled personnel to operate the machines 
among other factors (Barbarini et al., 2015). Additionally, in most African countries, standardized diagnostic methods and antibiotic panels for screening ESBLs, including carbapenemases, are not yet established (Rawat \& Najr, 2010). Hence, inadequate laboratory diagnosis and lack of surveillance suggests that CRE go undetected, untreated and might continue to spread unabated. In most cases, illnessess due to antibiotic resistant enteric bacteria are usually identified when treatment failure becomes recurrent (Okeke, 2016).

\section{CONCLUSION}

There is significant evidence to suggest that CRE is a major public health threat especially in Africa. Increasing carbapenem resistance and lack of more effective antibiotics beyond carbapenems suggests that a balance must be maintained between providing effective antibiotic treatment to patients who may die if not treated and preventing indiscriminate use of carbapenems. Due to epidemiological challenges such as variation in genetic makeup within and across countries, the detection and mitigation of the spread of carbapenem resistance amongst members of enterobacteriaceae has been challenging. With the paucity of data on CRE in Africa, studies using molecular techniques are urgently needed in the region. Ineffective detection methods which may lead to misdiagnosis and subsequent inappropriate antibiotic treatment, continues to promote the spread of CRE in hospital and community settings. Therefore, development of diagnostics that can detect AMR with high rapidity, sensitivity and specificity, at affordable prices is urgently needed in the developing world. In order to mitigate the impact of CRE, vaccines against enteric bacteria, new drug targets, prudent antimicrobial stewardship, and increased sensitization campaigns on appropriate use of antibiotics should be put in place across Africa.

\section{REFERENCES}

Barbarini, D., Russello, G., Brovarone, F., Capatti, C., Colla, R., Perilli, M., Moro, M.L., \& Caretto, E. (2015). Evaluation of carbapenem-resistant Enterobacteriaceae in an Italian setting: Report from the trench. Infection, Genetics and Evolution, 30, 8-14. doi:10.1016/j.meegid.2014.11.025

Bathoorn, E., Friedrich, A.W., Zhou, K., Arends, J.P., Borst, D.M., Grundmann, H., \& Rossen, J.W. (2013). Latent Introduction to the Netherlands of Multiple Antibiotic Resistance Including NDM-1 After Hospitalisation in Egypt, August 2013. Euro Surveillance, 18(42), 20610. doi:10.2807/1560-7917.es2013.18.42.20610

Brink, A., Coetzee, J., Clay, C., Corcoran, C., van Greune, J., Deetlefs, J.D., Nutt, L., Feldman, C., Richards, G., Nordmann, P., \& Poirel, L. (2012). South African Medical Journal, 102(7), 599-601. doi:10.7196/samj.5789

Chika, E., Malachy, U., Ifeanyichukwu, I.E., Peter, E., Thaddeus, G., \& Charles, E. (2014). Phenotypic Detection of Metallo- $\beta$-Lactamase ( MBL ) Enzyme in Enugu, Southeast Nigeria. American Journal of Biological, Chemical and Pharmaceutical Sciences, 2(2), 1-6.

Codjoe, F.S. \& Donkor, E.S. (2017). Carbapenem Resistance: A Review. Medical Sciences, 6(1), 1. doi:10.3390/medsci6010001

Codjoe, F.S., Donkor, E.S., Smith, T.J., \& Miller, K. (2019). Phenotypic and Genotypic Characterization of Carbapenem-Resistant Gram-Negative Bacilli Pathogens From Hospitals in Ghana. Microbial Drug Resistance, 25(10), 1449-1457. doi:10.1089/mdr.2018.0278

Dortet, L., Poirel, L., \& Nordmann, P. (2014). Worldwide Dissemination of the NDM-type Carbapenemases in Gram-negative Bacteria. BioMed Research International, 2014, 249856. doi:10.1155/2014/249856

Hawkey, P.M. \& Livermore, D.M. (2012). Carbapenem Antibiotics for Serious Infections. The BMJ, 344, 3236. doi:10.1136/bmj.e3236

Iovleva, A. \& Doi, Y. (2017). Carbapenem-Resistant Enterobacteriaceae. Clinics in Laboratory Medicine, 37(2), 303-315. doi:10.1016/j.cll.2017.01.005 
Kariuki, S. \& Dougan, G. (2014). Antibacterial resistance in sub-Saharan Africa: an underestimated emergency. Annals of the New York Academy of Sciences, 1323(1), 43-55. doi:10.1111/nyas.12380

Kelly, A.M., Mathema, B., Larson, E.L. (2017). Carbapenem-resistant Enterobacteriaceae in the Community: A Scoping Review. International Journal of Antimicrobial Agents, 50(2), 127-134. doi:10.1016/j.jijantimicag.2017.03.012

Kieffer, N., Nordmann, P., Aires-de-Sousa, M., \& Poirel, L. (2016). High Prevalence of CarbapenemaseProducing Enterobacteriaceae Among Hospitalized Children in Luanda, Angola. Antimicrobial Agents and Chemotherapy, 60(10), 6189-6192. doi:10.1128/aac.01201-16

Knapp, K.M. \& English, B.K. (2001). Carbapenems. Seminars in Pediatric Infectious Diseases, 12(3), 175-185. doi:10.1053/spid.2001.24093

Ktari, S., Arlet, G., Mnif, B., Gautier, V., Mahjoubi, F., Jmeaa, M.B., Bouaziz, M., \& Hammami, A. (2006). Emergence of Multidrug-Resistant Klebsiella Pneumoniae Isolates Producing VIM-4 Metallo-Beta-Lactamase, CTX-M-15 Extended-Spectrum Beta-Lactamase, and CMY-4 AmpC Beta-Lactamase in a Tunisian University Hospital. Antimicrobial Agents and Chemotherapy, 50(12), 4198-4201. doi:10.1128/aac.00663-06

Kumarasamy, K.K., Toleman, M.A., Walsh, T.R., Bagaria, J., Butt, F., Balakrishnan, R., Chaudhary, U., Coumith, M., Giske, C.G., Irfan, S., Khrisnan, P., Kumar, A.V., Maharjan, S., Mushtaq, S., Noorie, T., Paterson, D.L., Pearson, A., Perry, C., Pike, R., Rao, B., Ray, U., Sarma, J.B., Sharma, M., Sheridan, E., Thirunarayan, M.A., Turton, J., Upadhyay, S., Warner, M., Welfare, W., Livermore, D.M., \& Woodford, N. (2010). The Lancet Infectious Diseases, 10(9), 597-602. doi:10.1016/S1473-3099(10)70143-2

Lowman, W., Sriruttan, C., Nana, T., Bosman, N., Duse, A., Venturas, J., Clay, C., \& Coetzee, J. (2011). NDM-1 Has Arrived: First Report of a Carbapenem Resistance Mechanism in South Africa. South African Medical Journal, 101(12), 873-875.

Magiorakos, A.P., Burns, K., Rodriguez-Bano, J., Borg, M., Daikos, G., Dumpis, U., Lucet, J.C., Moro, M.L., Tacconelli, E., Skov-Simonsen, G.,
Szilagyi, E., Voss, A., \& Weber, J.T. (2017). Infection prevention and control measures and tools for the prevention of entry of carbapenem-resistant Enterobacteriaceae into healthcare settings: guidance from the European Centre for Disease Prevention and Control. Antimicrobial Resistance and Infection Control, 6, 113. doi:10.1186/s13756-017-0259-z

Manyi-Loh, C., Mamphweli, S., Meyer, E., \& Okoh, A. (2018). Antibiotic Use in Agriculture and Its Consequential Resistance in Environmental Sources: Potential Public Health Implications. Molecules, 23(4), 795. doi:10.3390/molecules23040795

Maphumulo, W.T. \& Bhengu, B.R. (2019). Challenges of quality improvement in the healthcare of South Africa post-apartheid: A critical review. Curationis, $\quad 42(1), \quad 1901$. doi:10.4102/curationis.v42i1.1901

Mitgang, E.A., Hartley, D.M., Malchione, M.D., Koch, M., \& Goodman, J.L. (2018). Review and Mapping of Carbapenem-Resistant Enterobacteriaceae in Africa: Using Diverse Data to Inform Surveillance Gaps. International Journal of Antimicrobial Agents, 52(3), 372-384. doi:10.1016/j.ijantimicag.2018.05.019

Moussounda, M., Diene, S.M., Dos Santos, S., Goudeau, A., Francois, P., van der Mee-Marquet, N. (2017). Emergence of Bla NDM-7-Producing Enterobacteriaceae in Gabon, 2016. Emerging Infectious Diseases, 23(2), 356-358. doi:10.3201/eid2302.161182

Muggeo, A., Guillard, T., Klein, F., Reffuveille, F., Francois, C., Babosan, A., Bajolet, O., Bertrand, X., de Champs, C., \& CarbaFrEst Group. Journal of Global Antimicrobial Resistance, 13, 98103. doi:10.1016/j.jgar.2017.10.023

Mushi, M.F., Mshana, S.E., Imirzalioglu, C., \& Bwanga, F. (2014). Carbapenemase Genes among Multidrug Resistant Gram Negative Clinical Isolates from a Tertiary Hospital in Mwanza, Tanzania. BioMed Research International, 2014, 303104. doi:10.1155/2014/303104

Ogbolu, D.O. \& Webber, M.A. (2014). High-level and Novel Mechanisms of Carbapenem Resistance in Gram-negative Bacteria From Tertiary Hospitals in Nigeria. International Journal of Antimicrobial Agents, 43(5), 412-417. doi:10.1016/j.ijantimicag.2014.01.014 
Okeke, I.N. (2016). Laboratory systems as an antibacterial resistance containment tool in Africa. African Journal of Laboratory Medicine, 5(3), 497. doi:10.4102/ajlm.v5i3.497

Okoche, D., Asiimwe, B.B., Katabazi, F.A., Kato, L., \& Najjuka, C.F. (2015). Prevalence and Characterization of Carbapenem-Resistant Enterobacteriaceae Isolated From Mulago National Referral Hospital, Uganda. PLoS One, 10(8), 0135745. doi:10.1371/journal.pone.0135745

Oladipo, E.K., Ajibade, O.A., Adeosun, I.J., Awoyelu, E.H., Akinade, S.B., Alabi, O.A., \& Ayilara, O.A. (2018). Antimicrobial resistance pattern of clinical isolates of Pseudomonas aeruginosa and Escherichia coli on carbapenems. African Journal of Clinical and Experimental Microbiology, 19(3), 159-164. doi:10.4314/ajcem.v19i3.1

Papp-Wallace, K.M., Endimiani, A., Taracila, M.A., \& Bonomo, R.A. (2011). Carbapenems: Past, Present, and Future. Antimicrobial Agents and Chemotherapy, 55(11), 4943-4960. doi:10.1128/AAC.00296-11

Perovic, O., Britz, E., Chetty, V., \& Singh-Moodley, A. (2016). Molecular detection of carbapenemaseproducing genes in referral Enterobacteriaceae in South Africa: A short report. South African Medical Journal, 106(10), 975-977. doi:10.7196/SAMJ.2016.v106i10.11300

Poirel, L., Revathi, G., Bernabeu, S., \& Nordmann, P. (2011). Detection of NDM-1-producing Klebsiella Pneumoniae in Kenya. Antimicrobial Agents and Chemotherapy, 55(2), 934-936. doi:10.1128/aac.01247-10

Rawat, D. \& Najr, D. (2010). Extended-spectrum $\beta$ lactamases in Gram Negative Bacteria. Journal of Global Infectious Diseases, 2(3), 263-274. doi:10.4103/0974-777X.68531

Robin, F., Aggoune-Khinache, N., Delmas, J., Naim, M., \& Bonnet, R. (2010). Novel VIM Metallo-BetaLactamase Variant From Clinical Isolates of Enterobacteriaceae From Algeria. Antimicrobial Agents and Chemotherapy, 54(1), 466-470. doi:10.1128/aac.00017-09

Rodriguez-Martinez, J.M., Nordmann, P., Fortineau, N., \& Poirel, L. (2010). VIM-19, a Metallo-BetaLactamase With Increased Carbapenemase Activity From Escherichia Coli and Klebsiella
Pneumoniae. Antimicrobial Agents and Chemotherapy, 54(1), 471-476. doi:10.1128/aac.00458-09

Sangare, S.A., Rondinaud, E., Maataoui, N., Maiga, A.I., Guindo, I., Maiga, A., Camara, N., Dicko, O.A., Dao, S., Diallo, S., Bougoudogo, F., Andremont, A., Maiga, I.I., Armand-Lefevre, L. (2017). Very High Prevalence of ExtendedSpectrum Beta-Lactamase-Producing Enterobacteriaceae in Bacteriemic Patients Hospitalized in Teaching Hospitals in Bamako, Mali. PLoS One, 12(2), 0172652. doi:10.1371/journal.pone.0172652

Sekyere, J.O., Govinden, U., \& Essack, S. (2016). The Molecular Epidemiology and Genetic Environment of Carbapenemases Detected in Africa. Microbial Drug Resistance, 22(1), 59-68. doi:10.1089/mdr.2015.0053

Tadesse, B.T., Ashley, E.A., Ongarello, S., Havumaki, J., Wijegoonewardena, M., Gonzales, I.J., \& Dittrich, S. (2017). Antimicrobial Resistance in Africa: A Systematic Review. BMC Infectious Diseases, 17(1), 616. doi:10.1186/s12879-0172713-1

Tischendorf, J., de Avila, R.A., \& Safdar, N. (2016). Risk of Infection Following Colonization With Carbapenem-Resistant Enterobactericeae: A Systematic Review. American Journal of Infection Control, 44(5), 539-543. doi:10.1016/j.ajic.2015.12.005

Ventola, C.L. (2015). The Antibiotic Resistance Crisis: Part 1: Causes and Threats. Pharmacy and Therapeutics, 40(4), 277-283.

Yusuf, I., Yusha'u, M., Sharif, A.A., Getso, M.I., Yahaya, H., Bala, J.A., Aliyu, I.A., \& Haruna, M. (2012). Detection of metallo betalactamases among gram negative bacterial isolates from Murtala Muhammad Specialist Hospital, Kano and Almadina Hospital Kaduna, Nigeria. Bayero Journal of Pure and Applied Sciences, 5(2), 84-88. doi:10.4314/bajopas.v5i2.15

Zhanel, G.G., Wiebe, R., Dilay, L., Thomson, K., Rubinstein, E., Hoban, D.J., Noreddin, A.M., \& Karlowsky, J.A. (2007). Comparative Review of the Carbapenems. Drugs, 67(7), 1027-1052. doi:10.2165/00003495-200767070-00006 